\title{
Phase Separation of Binary Systems
}

\author{
Tian Ma \\ Department of Mathematics, Sichuan University, Chengdu, P. R. China \\ Shouhong Wang* \\ Department of Mathematics, Indiana University, Bloomington, IN $47405{ }^{\dagger}$
}

(Dated: June 16, 2021)

\begin{abstract}
In this Letter, three physical predictions on the phase separation of binary systems are derived based on a dynamic transition theory developed recently by the authors. First, the order of phase transitions is precisely determined by the sign of a parameter $K_{d}$ (or a nondimensional parameter $K$ ) such that if $K_{d}>0$, the transition is first-order with latent heat and if $K_{d}<0$, the transition is second-order. Second, a theoretical transition diagram is derived, leading in particular to a prediction that there is only second-order transition for molar fraction near $1 / 2$. This is different from the prediction made by the classical transition diagram. Third, a critical length scale $L_{d}^{c}$ is derived such that no phase separation occurs at any temperature if the length of the container is smaller than the critical length scale.
\end{abstract}

PACS numbers: 05.70.Fh, 64.60.Ht

Keywords: binary system, Cahn-Hilliard equation, phase diagram, order of separation, critical length scale, dynamic transition theory

Materials compounded by two components $A$ and $B$, such as binary alloys, binary solutions and polymers, are called binary systems. Sufficient cooling of a binary system may lead to phase separations, i.e., at the critical temperature, the concentrations of both components $A$ and $B$ with homogeneous distribution undergo changes, leading to heterogeneous spatial distributions. The main objective of this Letter is to precisely describe the phase separation mechanism and to make a few physical predictions.

Cahn-Hilliard Equation. Let $u_{A}$ and $u_{B}$ be the concentrations of components $A$ and $B$ respectively, then $u_{B}=1-u_{A}$. In a homogeneous state, $u_{B}=\bar{u}_{B}$ is a constant. We take $u$ to be the concentration density deviation $u=u_{B}-\bar{u}_{B}$. The Cahn-Hilliard free energy is given by

$$
F(u)=F_{0}+\int_{\Omega}\left[\frac{\mu}{2}|\nabla u|^{2}+f(u)\right] d x,
$$

where

$$
f(u)=\alpha_{1} u^{2}+\alpha_{2} u^{3}+\alpha_{3} u^{4} .
$$

The same results in this article can be derived in the same fashion, and for simplicity, we take this form of $f$ as given here. Then the classical Cahn-Hilliard equation is as follows:

$$
\begin{aligned}
& \frac{\partial u}{\partial t}=-k \Delta^{2} u+\Delta\left[b_{1} u^{1}+b_{2} u^{2}+b_{3} u^{3}\right], \\
& \int_{\Omega} u(x, t) d x=0,
\end{aligned}
$$

*URL: http://www.indiana.edu/ fluid

${ }^{\dagger}$ This work is supported in part by grants from ONR and NSF. supplemented with the Neumann boundary condition:

$$
\frac{\partial u}{\partial n}=\frac{\partial \Delta u}{\partial n}=0 \quad \text { on } \partial \Omega
$$

where $\Omega=\Pi_{k=1}^{3}\left(0, L_{k}\right) \subset \mathbb{R}^{3}$ is a rectangular domain. We note that the more general domain case can be studied as well.

To derive the nondimensional form of equation, let

$$
\begin{array}{lll}
x=l x^{\prime}, & t=\frac{l^{4}}{k} t^{\prime}, & u=u_{0} u^{\prime}, \\
\lambda=-\frac{l^{2} b_{1}}{k}, & \gamma_{2}=\frac{l^{2} b_{2} u_{0}}{k}, & \gamma_{3}=\frac{l^{2} b_{3} u_{0}^{2}}{k},
\end{array}
$$

where $l$ is a given length, $u_{0}=\bar{u}_{B}$ is the constant concentration of $B$, and $\gamma_{3}>0$. Then the equation (2) can be rewritten as follows (omitting the primes)

$$
\begin{aligned}
& \frac{\partial u}{\partial t}=-\Delta^{2} u-\lambda \Delta u+\Delta\left(\gamma_{2} u^{2}+\gamma_{3} u^{3}\right), \\
& \int_{\Omega} u(x, t) d x=0, \\
& u(x, 0)=\varphi .
\end{aligned}
$$

Criteria of separation order. Each 3D rectangular domain is one of the following two cases:

$$
\begin{array}{lc}
\text { Case I: } & L=L_{1}>L_{j} \quad \forall j \leq 2,3, \\
\text { Case II: } & L=L_{1}=L_{2}>L_{3} \text { or } L_{1}=L_{2}=L_{3} .
\end{array}
$$

We define a nondimensional parameter:

$$
K= \begin{cases}\frac{2 L^{2}}{9 \pi^{2}} \gamma_{2}^{2}-\gamma_{3} & \text { for Case I, } \\ \frac{26 L^{2}}{27 \pi^{2}} \gamma_{2}^{2}-\gamma_{3} & \text { for Case II. }\end{cases}
$$


which, by (4), is equivalent to the following dimensional parameter:

$$
K_{d}= \begin{cases}\frac{2 L_{d}^{2}}{9 \pi^{2}} \frac{b_{2}^{2}}{k}-b_{3} & \text { for Case I, } \\ \frac{26 L_{d}^{2}}{27 \pi^{2}} \frac{b_{2}^{2}}{k}-b_{3} & \text { for Case II. }\end{cases}
$$

where $L_{d}=L \cdot l$ is the dimensional length scale.

By theorems proved in 4, the order of transitions is determined by the sign of this parameter $K$ or $K_{d}$ as follows, and we have readily derived the following physical predictions:

Physical Conclusion I: The order of phase separation is completely determined by the sign of the nondimensional parameter $K_{d}$ as follows:

(1) If $K_{d}<0$, the separation is second order and the dynamic behavior of the Cahn-Hilliard system is as shown in Figure 1.

(2) If $K_{d}>0$, the separation is first order transition with latent heat. In particular, there are two critical temperature $T^{*}>T_{c}$ such that if the temperature $T>T^{*}$, the system is in the homogeneous state, when $T^{*}>T>T_{c}$, the system is in metastable state accompanied with hysteresis corresponding to saddle-node bifurcation, and when $T<T_{c}$, the system is under phase separation state. In addition, the critical temperatures are functions of $u_{0}$ and $L$ : $T^{*}=T^{*}\left(u_{0}, L\right), T_{c}=T_{c}\left(u_{0}, L\right)$. See Figure 2.

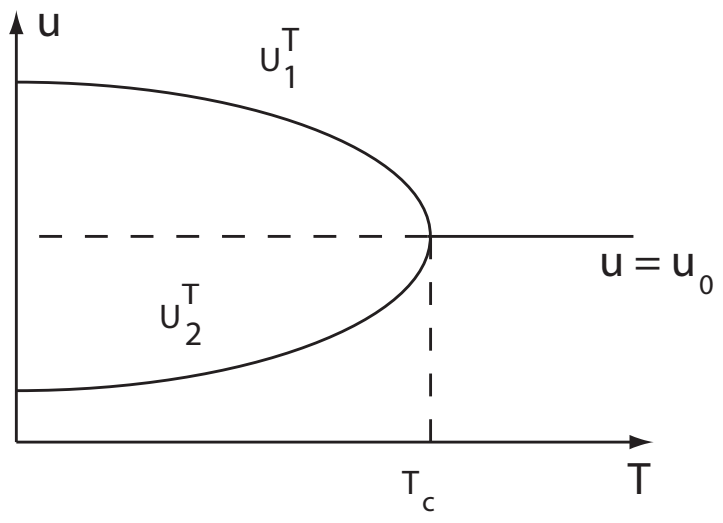

FIG. 1: The state $u_{0}=\bar{u}_{B}$ is stable if $T_{c}<T$, and the state $u_{0}$ is unstable, $U_{1}^{T}$ and $U_{2}^{T}$ are stable if $T<T_{c}$.

This is in agreement with part of the classical phase diagram from the classical thermodynamic theory given in Figure 3, see, among others, Reichl [8], Novick-Cohen and Segal [7], and Langer [1. However, as we shall see below, our result shows that near $u_{0}=1 / 2$, there is no metastable region; see Figure 6 .

Transition diagram. We now examine the order of separation in terms of the length scale $L_{d}$ and mol fraction $u_{0}$. For this purpose, according to the Hildebrand

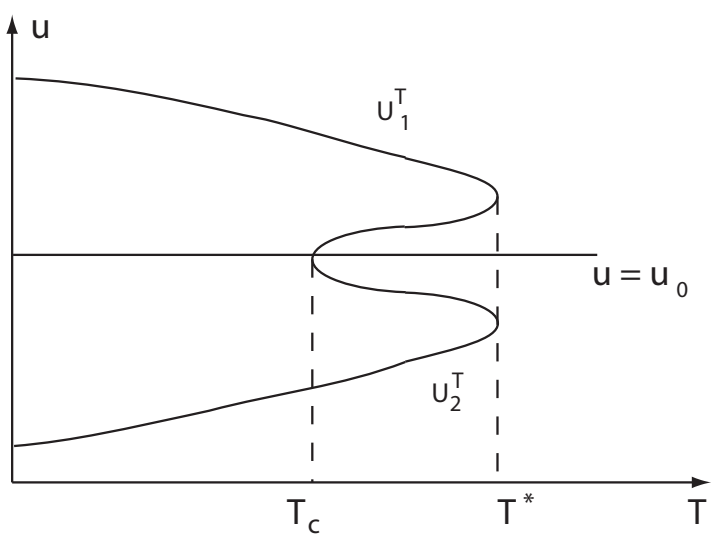

FIG. 2: For fixed $u_{0}$ and $L$, the transition for the case where $K_{d}>0$ is first order separation with latent heat and with hysteresis: $U_{1}^{T}$ and $U_{2}^{T}$ represent separation states, and $u_{0}$ is the homogeneous state. In this case, for $T_{c}<T<T^{*}$, all states $u_{0}, U_{1}^{T}, u_{2}^{T}$ are metastable states. For $T<T_{c}, u_{0}$ is unstable, and $U_{1}^{T}$ and $U_{2}^{T}$ are stable states.

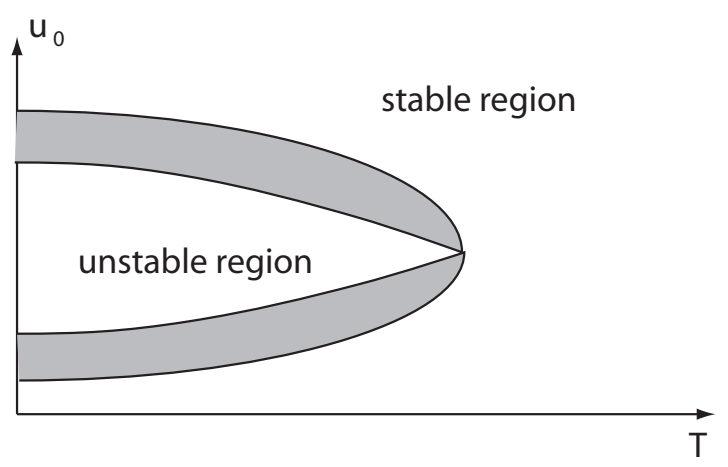

FIG. 3: Typical phase diagram from classical thermodynamic theory with shadowed being the metastable region .

theory (see Reichl [8]), $b_{2}$ and $b_{3}$ can be expressed in two explicit formulas. Disregarding the term $|\nabla u|^{2}$, the molar Gibbs free energy takes the following form

$$
\begin{aligned}
f= & \mu_{A}(1-u)+\mu_{B} u+R T(1-u) \ln (1-u) \\
& +R T u \ln u+a u(1-u)
\end{aligned}
$$

where $\mu_{A}, \mu_{B}$ are the chemical potential of $A$ and $B$ respectively, $R$ the molar gas constant, $a>0$ the measure of repel action between $A$ and $B$. Therefore, the coefficients $b_{2}$ and $b_{3}$ are given by

$$
\begin{aligned}
& b_{2}=\frac{D}{3 !} \frac{d^{3} f\left(u_{0}\right)}{d u^{3}}=\frac{2 u_{0}-1}{6 u_{0}^{2}\left(1-u_{0}\right)^{2}} D R T, \\
& b_{3}=\frac{D}{4 !} \frac{d^{4} f\left(u_{0}\right)}{d u^{4}}=\frac{1-3 u_{0}+3 u_{0}^{2}}{12 u_{0}^{3}\left(1-u_{0}\right)^{3}} D R T,
\end{aligned}
$$


where $D$ is the diffusion coefficient. It is easy to see that

$$
\begin{aligned}
& b_{2} \begin{cases}=0 & \text { if } u_{0}=\frac{1}{2}, \\
\neq 0 & \text { if } u_{0} \neq \frac{1}{2},\end{cases} \\
& b_{3}>0 \quad \forall 0<u_{0}<1 .
\end{aligned}
$$

It is clear that the above formulas for $b_{2}$ and $b_{3}$ based on the Hildebrand theory fail near $u_{0}=0,1$. However, the physically relevant case is away from these two end points of $u_{0}$, and then we have:

$$
\begin{aligned}
& b_{2}=\frac{16 D R T}{3}\left(u_{0}-\frac{1}{2}\right)+o\left(u_{0}-\frac{1}{2}\right), \\
& b_{3}=\frac{4 D R T}{3}+o(1) .
\end{aligned}
$$

Then solving $K_{d}=0$ gives a critical (dimensional) length scale $L_{d}$ :

$$
L_{d}= \begin{cases}\frac{3 \pi \sqrt{k}}{\sqrt{2}} \frac{\sqrt{b_{3}}}{\left|b_{2}\right|} & \text { for Case I, } \\ \frac{3 \pi \sqrt{3 k}}{\sqrt{26}} \frac{\sqrt{b_{3}}}{\left|b_{2}\right|} & \text { for Case II. }\end{cases}
$$

By (10) and 111), we have

$$
L_{d}= \begin{cases}\frac{3 \sqrt{3 k} \pi}{8 \sqrt{2 D R T_{c}}\left|u_{0}-\frac{1}{2}\right|}+O(1) & \text { for Case I, } \\ \frac{9 \sqrt{k} \pi}{8 \sqrt{26 D R T_{c}}\left|u_{0}-\frac{1}{2}\right|}+O(1) & \text { for Case II, }\end{cases}
$$

where $T_{c}$ is the critical temperature as given in Physical Conclusion I. From this formula, we derive the transition diagram given by Figure 4, and consequently, we derive a theoretical phase diagram given in Figure 5. In particular, we have shown the following physical conclusions:

\section{Physical Conclusion II.}

(1) For a fixed length scale $L=L^{\prime}$, there are numbers $x_{1}<\frac{1}{2}<x_{2}$ such that the transition is secondorder if the molar fraction $x_{1}<u_{0}<x_{2}$, and the transition is first-order if $u_{0}>x_{2}$ or $u_{0}<x_{1}$.

(2) The phase diagram Figure 5 is for this fixed length scale $L^{\prime}$. The points $x_{1}$ and $x_{2}$ are the two molar concentrations where there is no metastable region and no hysteresis phenomena for $x_{1}<u_{0}<x_{2}$. In other words,

$$
T^{*}\left(u_{0}\right)=T_{c}\left(u_{0}\right) \quad \text { for } x_{1}<u_{0}<x_{2} .
$$

$T L$-phase diagram. We now derive the length and temperature phase diagram. For this purpose, we consider the linear eigenvalue problem for the Cahn-Hilliard equation as follows:

$$
\begin{aligned}
& -\Delta^{2} u-\lambda \Delta u=\beta u, \\
& \frac{\partial u}{\partial n}=\frac{\partial \Delta u}{\partial n}=0 \quad \text { on } \partial \Omega .
\end{aligned}
$$

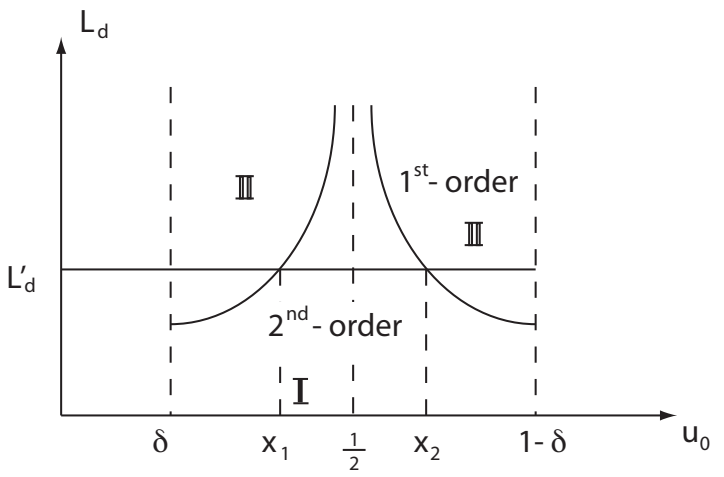

FIG. 4: Transition diagram: region II is the first order transition region with latent heat, and region $I$ is the second order transition region.

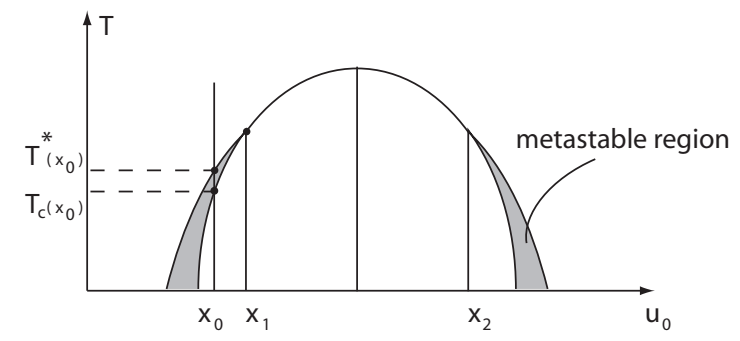

FIG. 5: Derived theoretical diagram for a fixed length scale $L^{\prime}$ with the shadowed region being the metastable region.

The first eigenvalue is given by

$$
\beta_{1}=-\frac{\pi^{2}}{L^{2}}\left(\frac{\pi^{2}}{L^{2}}-\lambda\right)=-\frac{\pi^{2}}{L^{2}}\left(\frac{\pi^{2}}{L^{2}}+\frac{l^{2} b_{1}}{k}\right) .
$$

By (10), we have

$$
b_{1}=\frac{D}{2} \frac{d^{2} f\left(u_{0}\right)}{d u^{2}}=\frac{D R T}{2 u_{0}\left(1-u_{0}\right)}-\frac{a}{2} .
$$

The critical parameter curve equation $\beta_{1}=0$ is given by

$$
\begin{aligned}
T_{c} & =\frac{u_{0}\left(1-u_{0}\right)}{R D}\left(a-\frac{k \pi^{2}}{2 l^{2} L^{2}}\right) \\
& =\frac{u_{0}\left(1-u_{0}\right)}{R D}\left(a-\frac{k \pi^{2}}{2 L_{d}^{2}}\right) .
\end{aligned}
$$

Using this formula and the theorems in [4], we derive the $T L$ phase diagram given by Figure 6, and the following physical conclusions:

Physical Conclusion III. For a given molar fraction $0<u_{0}<1$, there is a critical (dimensional) length

$$
L_{d}^{c}=\sqrt{\frac{k \pi^{2}}{2 a}}
$$

such that the following hold true:

(1) For $L_{d}<L_{d}^{c}$, there is no phase separation for any temperature. 


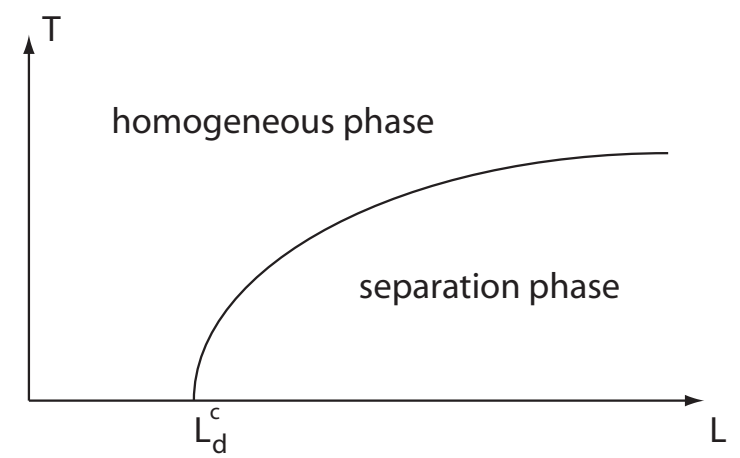

FIG. 6: TL phase diagram.

(2) For $L_{d}>L_{d}^{c}$, phase separation occurs at the critical temperature $T=T_{c}$ given by (14).

Summary. Based on a dynamic transition theory developed recently by the authors [2, 3, 5, 6, , a systematic mathematical analysis is made for the Cahn-Hilliard equation modeling phase separation of binary systems 4]. Based on this rigorous analysis, we are able to make three physical predictions on the phase separation of binary systems:

FIRST, the order of phase transitions is precisely de- termined by the sign of a parameter $K_{d}$ (or a nondimensional parameter $K$ ) such that if $K_{d}>0$, the transition is first-order with latent heat and if $K_{d}<0$, the transition is second-order. This parameter $K_{d}$ is explicitly given in terms of the system properties and the geometry of the container.

SECOND, a theoretical transition diagram is derived, leading in particular to a prediction that there is only second-order transition for molar fraction near $1 / 2$. This is different from the prediction made by the classical transition diagram.

THIRD, a critical length scale $L_{d}^{c}$ is derived such that no phase separation even occurs at any temperature if the length scale of the container is smaller than the critical length scale. The transition temperature $T_{c}$ is precisely given as well for the length scale is larger than the critical scale.

FinALLY, our theory fully reveals the transition dynamics. This is the advantage of using the dynamic classification scheme as proposed in [3, 5, 6, where the transitions are classified as Type-I, Type-II and TypeIII. Also, we would like to mention that our results are derived for rectangular domains, and more general domain case can be studied using the dynamic transition theory as well, and other transition types such as the mixed transition may occur; see [4].
[1] J. LANGER, Theory of spinodal decomposition in allays, Ann. of Physics, 65 (1971), pp. 53-86.

[2] T. MA AND S. WANG, Bifurcation theory and applications, vol. 53 of World Scientific Series on Nonlinear Science. Series A: Monographs and Treatises, World Scientific Publishing Co. Pte. Ltd., Hackensack, NJ, 2005.

[3] — Stability and Bifurcation of Nonlinear Evolutions Equations, Science Press (in Chinese), Beijing, 2007.

[4] —, Cahn-hilliard equations and phase transition dynamics for binary systems, Dist. Cont. Dyn. Systs., Ser. B, (2008); see also arXiv:0806.1286.

[5] - Dynamic phase transition theory in PVT systems,
Indiana University Mathematics Journal, to appear; see also Arxiv: 0712.3713, (2008).

[6] — Dynamic phase transitions for ferromagnetic systems, Journal of Mathematical Physics, 49:053506 (2008), pp. $1-18$.

[7] A. Novick-Cohen And L. A. Segel, Nonlinear aspects of the Cahn-Hilliard equation, Phys. D, 10 (1984), pp. 277298.

[8] L. E. REICHL, A modern course in statistical physics, A Wiley-Interscience Publication, John Wiley \& Sons Inc., New York, second ed., 1998. 